\title{
An Examination of the Effects of Offshore and Outsourced Development on the Delegation of Responsibilities to Software Components
}

\author{
Subhajit Datta ${ }^{\star}$ and Robert van Engelen \\ Department of Computer Science and School of Computational Science, Florida State \\ University, Tallahassee, FL 32306, USA \\ sd05@fsu.edu
}

\begin{abstract}
Offshore and outsourced development are the latest facts of life of professional software building. The easily identifiable advantages of these trends - such as cost benefits, continuous delivery and support - have already been explored to considerable extent. But how does offshore and outsourced development affect the delegation of responsibilities to components of a software system? In this paper we investigate this question by applying the RESP-DIST technique on a set of real life case studies. Our RESP-DIST technique uses metrics and a linear programming based method to recommend the reorganization of components towards an expedient distribution of responsibilities. The case studies embody varying degrees of offshore and outsourced development. Results from the case studies lead to some interesting observations on whether and how offshore and outsourced development influences software design characteristics.
\end{abstract}

\section{Introduction}

The paradigm of offshore and outsourced software development involves distribution of life cycle activities and stakeholder interests across geographical, political, and cultural boundaries. In this paper we will use the phrase dispersed development to refer to offshore and outsourced software development. We use the term "dispersed" in the sense of distribution of software development resources and concerns across different directions and wide area.

We seek to examine whether dispersed development has any impact on how responsibilities are delegated to components in a software system. Towards this end, we will apply the RESP-DIST technique across a spectrum of software development projects and analyze the results. RESP-DIST is a mechanism to guide RESPonsibility DISTribution in components of a software system such that they are best able to collectively deliver the system's functionality. RESPDIST uses the metrics Mutation Index 6, Aptitude Index, and Concordance Index [7] and a linear programming (LP) based algorithm [7] to recommend the merging or splitting (as defined in more detail in the next section) of components

\footnotetext{
^ Corresponding author.

K. Berkling et al. (Eds.): SEAFOOD 2008, LNBIP 16, pp. 73-89 2009.

(C) Springer-Verlag Berlin Heidelberg 2009
} 
to lead to an even distribution of responsibilities and resilience to requirement changes. As discussed in a later section, aptitude and concordance are the design characteristics which RESP-DIST considers while making its recommendations. By applying RESP-DIST across a set of software projects of varying dispersion in their development, we expect to discern whether and how offshore and outsourced development affects the responsibility delegation aspect of software design.

The remainder of this paper is organized as follows: In the next section we describe a model for the software development space which will serve as a foundation for applying RESP-DIST. The following section informally reviews some key concerns of software design, followed by the introduction of the ideas of aptitude and concordance. We then define the metrics and specify the RESP-DIST technique. Subsequently, results from applying RESP-DIST on five real life software projects are presented. The paper ends with a discussion of related work, open issues and planned future work, and conclusions.

\section{A Model for the Software Development Space}

The discussion of this paper is based on the following connotations of certain definitions:

- A requirement is described as "... a design feature, property, or behaviour of a system" by Booch, Rumbaugh, and Jacobson 2. These authors refer to the statement of a system's requirements as the assertion of a contract on what the system is expected to do; how the system does that is essentially for the designer to decide.

- A component carries out specific responsibilities and interacts with other components through its interfaces to collectively deliver the system's functionality (within acceptable non-functional parameters).

- A collaboration is described in the Unified Modelling Language Reference Manual, Second Edition as a "... society of cooperating objects assembled to carry out some purpose" [18. Components collaborate via messages to fulfil their tasks. In this paper "collaboration" and "interaction" will be used interchangeably.

- Merging of a particular component will be taken to mean distributing its responsibilities to other components in the system and removing the component from the set of components fulfilling a given set of requirements. So after merging, a set of components will be reduced in number, but will be fulfilling the same set of requirements as before.

- Splitting of a particular component will be taken to mean distributing some its responsibilities to a new component in the system which will interact on its own with other components to collectively deliver the system's functionality. So after splitting, a set of components will be increased in number, but will be fulfilling the same set of requirements as before.

We now present an abstraction of how requirements are fulfilled by components. 
In order to examine the dynamics of software systems through a set of metrics, a model is needed to abstract the essential elements of interest 7 .

Let the development space of a software system consist of the set requirements $R e q=\left\{R_{1}, \ldots, R_{x}\right\}$ of the system, which are fulfilled by the set of components Comp $=\left\{C_{1}, \ldots, C_{y}\right\}$.

We take fulfilment to be the satisfaction of any user defined criteria to judge whether a requirement has been implemented. Fulfilment involves delivering the functionality represented by a requirement. A set of mapping exists between requirements and components, we will call these relationships. At one end of a relationship is a requirement, at the other ends are all the components needed to fulfil it. Requirements also mesh with one another - some requirements are linked to other requirements, as all of them belong to the same system, and collectively specify the overall scope of the system's functionality. The links between requirements are referred to as connections. From the designer's point of view, of most interest is the interplay of components. To fulfil requirements, components need to collaborate in some useful ways, these are referred to as the interactions of components.

Based on this model, an important goal of software design can be stated as: Given a set of connected requirements, how to devise a set of interacting components, such that the requirements and components are able to forge relationships that best deliver the system's functionality within given constraints?

\section{Key Concerns of Software Design}

To examine whether and how distributed development affects software design, we discuss the act of design in some detail.

We may say that the conception of a particular system's design is instantiated by allocating particular tasks to components and specifying their interaction with other components such that the set of components collectively fulfil the system's requirements within acceptable non-functional parameters such as performance etc. In this paper, we focus entirely on the functional aspect of design. How does one decide on allocating tasks and specifying interactions?

Larman has called the ability to assign responsibilities as a "desert-island skill" [16, to underline its criticality in the design process. Indeed, deciding which component does what remains a key challenge for the software designer. Ideally, each component should perform a specialized task and cooperate with other components to deliver the system's overall functionality. Whenever a new functionality comes to light by analyzing (new or modified) requirements, the designer's instinct is to spawn a new component and assign it the task for delivering that functionality. This new component acts as something of an initial placeholder for the new functionality; to be reconsidered later if necessary. With increasing accretion of functionality a system usually ends up having a large number of fine grained components. Why does the designer instinctively spawn a new component for a new functionality, and not just commandeer an existing component to deliver that functionality? 
The instinct perhaps is inspired by one of the lasting credos of effective and elegant software design: shunning large, bloated units of code (the so called "Swiss army knife" or "do-it-all" components) in preference to smaller, more coherent, and closely collaborative ones. However, recognizing the inherently iterative nature of software design, there is always scope - sometimes a pressing need for deciding to merge some components while splitting others as development proceeds. Merging helps consolidate related responsibilities, thereby decreasing redundant and sometimes costly method calls across components. It is a natural way to refine components and their interactions after new components had been spawned (often indiscriminately) earlier to address new functionalities. But often, splitting a component is an useful way to isolate the implementation of a piece of functionality that is undergoing frequent modifications due to changing requirements. Such isolation helps insulate other components and their interactions from the effects of requirements that change often.

Thus the design of a software system in terms of its components - their individual responsibilities and collective interaction - matures iteratively through merging and splitting. Over such repeated reorganizations, design objectives of expediently fulfilling requirements as well as being resilient to some of their changes, are progressively met. But how does one decide on which component to merge and which to split? This is one the most important concerns of the software designer, usually addressed through experience or intuition or nameless "gut-feelings". RESP-DIST brings in a degree of discipline and sensitivity into such decisions - the technique seeks to complement the best of designers' judgment, and constrict their worst. RESP-DIST leverages certain characteristics of a software system's design which we discuss next.

\section{Delegation of Responsibilities in Software Design}

Design is usually an overloaded word, even in the software development context. There are no universally accepted features of good design, while symptoms of bad design are easy to discern. In the model for the software development space presented in an earlier section, we highlighted one aspect of the design problem. Based on this aspect, we introduce aptitude and concordance [7] as two key characteristics of design.

Every software component exists to perform specific tasks, which may be called its responsibilities. Software design canons recommend that each component be entrusted with one primary responsibility. In practice, components may end up being given more than one task, but it is important to try and ensure they have one primary responsibility. Whether components have one or more responsibilities, they can not perform their tasks entirely by themselves, without any interaction with other components. This is specially true for the so-called business objects - components containing the business logic of an application. The extent to which a component has to interact with other components to fulfil its core functionality is an important consideration. If a component's responsibilities are strongly focused on a particular line of functionality, its interactions 
with other components can be expected to be less disparate. We take aptitude to denote the quality of a component that reflects how coherent its responsibilities are. Intuitively, the Aptitude Index measures the extent to which a component (one among a set fulfilling a system's requirements) is coherent in terms of the various tasks it is expected to perform.

The Aptitude Index [7] seeks to measure how coherent a component is in terms of its responsibilities.

To each component $C_{m}$ of Comp, we attach the following properties [5]. A property is a set of zero, one or more components.

- Core - $\alpha(m)$

- Non-core - $\beta(m)$

- Adjunct - $\gamma(m)$

$\alpha(m)$ represents the set of component(s) required to fulfil the primary responsibility of the component $C_{m}$. As already noted, sound design principles suggest the component itself should be in charge of its main function. Thus, most often $\alpha(m)=\left\{C_{m}\right\}$.

$\beta(m)$ represents the set of component(s) required to fulfil the secondary responsibilities of the component $C_{m}$. Such tasks may include utilities for accessing a database, date or currency calculations, logging, exception handling etc.

$\gamma(m)$ represents the component(s) that guide any conditional behaviour of the component $C_{m}$. For example, for a component which calculates interest rates for bank customers with the proviso that rates may vary according to a customer type ("gold", "silver" etc.), an Adjunct would be the set of components that help determine a customer's type.

Definition 1. The Aptitude Index $A I(m)$ for a component $C_{m}$ is a relative measure of how much $C_{m}$ depends on the interaction with other components for delivering its core functionality. It is the ratio of the number of components in $\alpha(m)$ to the sum of the number of components in $\alpha(m), \beta(m)$, and $\gamma(m)$.

$$
A I(m)=\frac{|\alpha(m)|}{|\alpha(m)|+|\beta(m)|+|\gamma(m)|}
$$

As reflected upon earlier, the essence of software design lies in the collaboration of components to collectively deliver a system's functionality within given constraints. While it is important to consider the responsibility of individual components, it is also imperative that inter-component interaction be clearly understood. Software components need to work together in a spirit of harmony if they have to fulfil requirements through the best utilization of resources. Let us take concordance to denote such cooperation amongst components. How do we recognize such cooperation? It is manifested in the ways components share the different tasks associated with fulfilling a requirement. Some of the symptoms of less than desirable cooperation are replication of functionality - different components doing the same task for different contexts, components not honouring their interfaces (with other components) in the tasks they perform, one component 
trying to do everything by itself etc. The idea of concordance is an antithesis to all such undesirable characteristics - it is the quality which delegates the functionality of a system across its set of components in a way such that it is evenly distributed, and each task goes to the component most well positioned to carry it out. Intuitively, the metric Concordance Index [7] measures the extent to which a component is concordant in relation to its peer components in the system.

Definition 2. The Concordance Index $C I(m)$ for a component $C_{m}$ is a relative measure of the level of concordance between the requirements being fulfilled by $C_{m}$ and those being fulfilled by other components of the same system.

The Requirement Set $R S(m)$ for a component $C_{m}$ is the set of requirements that need $C_{m}$ for their fulfilment 7 .

For a set of components $C o m p=\left\{C_{1}, C_{2}, \ldots, C_{n}, \ldots, C_{y-1}, C_{y}\right\}$ let, $W=R S(1) \cup R S(2) \cup \ldots \cup R S(y-1) \cup R S(y)$

For a component $C_{m}(1 \leq m \leq y)$, let us define, $X(m)=(R S(1) \cap R S(m)) \cup \ldots \cup((R S(m-1) \cap R S(m)) \cup$ $((R S(m) \cap(R S(m+1)) \cup \ldots \cup((R S(m) \cap(R S(y))$

Thus $X(m)$ denotes the set of requirements that are not only being fulfilled by $C_{m}$ but also by some other component(s).

Expressed as a ratio, the Concordance Index $C I(m)$ for component $C_{m}$ is:

$$
C I(m)=\frac{|X(m)|}{|W|}
$$

How do the ideas of aptitude and concordance relate to cohesion and coupling? Cohesion is variously defined as " ... software property that binds together the various statements and other smaller modules comprising the module" 8 and "... attribute of a software unit or module that refers to the relatedness of module components" [1]. (In the latter quote, "component" has been used in the sense of part of a whole, rather than a unit of software as is its usual meaning in this paper.) Thus cohesion is predominantly an intra-component idea pointing to some feature of a module that closely relates its constituents to one another. But as discussed above, concordance carries the notion of concord or harmony, signifying the spirit of successful collaboration amongst components towards collective fulfilment of a system's requirements. Concordance is an intercomponent idea; the concordance of a component can only be seen in the light of its interaction with other components.

Coupling has been defined as " $\ldots$ a measure of the interdependence between two software modules. It is an intermodule property" 88. Thus coupling does not take into account the reasons for the so called "interdependence" - that modules (or components) need to cooperate with one another as they must together fulfil a set of connected requirements. Aptitude is an intra-component idea, which reflects on a component's need to rely on other components to fulfil its primary responsibility/responsibilities. 
Cohesion and coupling are legacy ideas from the time when software systems were predominantly monolithic. In the age of distributed systems, successful software is built by carefully regulating the interaction of components, each of which are entrusted with clearly defined responsibilities. The perspectives of aptitude, and concordance complement cohesion and coupling in helping recognize, isolate, and guide design choices that will lead to the development of usable, reliable, and evolvable software systems.

As mentioned earlier, one of the main drivers of design change is changing requirements. Let us take the term mutation to mean any change in a particular requirement that would require a modification in one or more components fulfilling either one or a combination of the display, processing, or storage aspects of the requirement. In keeping with the principle of separation of concerns, it is usually taken to be good design practice to assign specific components to deliver each of the display, processing, and storage aspects. Components (or sets of components) delegated to fulfil the display, processing, and storage aspects of requirement(s) map to the stereotypes of analysis classes: boundary, control, and entity [13. Intuitively, the metric Mutation Index [6] measures the extent to which a requirement has changed from one iteration to another, in terms of its display, processing, and storage aspects.

For a system let $R e q=\left\{R_{1}, R_{2}, \ldots, R_{m} \ldots, R_{x}\right\}$ denote the set of requirements. Between iterations $I_{z-1}$ and $I_{z}$ each requirement is annotated with its Mutation Value; a combination of the symbols $D, P$ and $S$. The symbols stand for:

$D \equiv \operatorname{Display}(1)$

$P \equiv$ Processing $(3)$

$S \equiv$ Storage $(2)$

The parenthesized numbers denote the Weights attached to each symbol. The combination of more than one symbol signifies the addition of their respective Weights, thus:

$P D \equiv D P \equiv 1+3=4$

$S D \equiv D S \equiv 1+2=3$

$S P \equiv P S \equiv 3+2=5$

$S P D \equiv \ldots \equiv D P S \equiv 1+3+2=6$

The Weight assigned to each category of components - Display, Processing and Storage - is a relative measure of their complexities. (Complexity here refers to how intense the design, implementation, and maintenance of a component are in terms of development effort.) Processing components usually embody application logic and are most design and implementation intensive. Storage components encapsulate the access and updating of application data stores; their level of complexity is usually lower than that of the Processing components but higher than Display ones. Accordingly, Display, Processing and Storage have been assigned the Weights 1, 3 and 2 respectively. Exact values of Weights may be varied from one project to another; the essential idea is to introduce a quantitative differentiation between the types of components. 
Definition 3. The Mutation Index $M I(m)$ for a requirement $R_{m}$ is a relative measure of the extent to which the requirement has changed from one iteration to another in terms of the components needed to fulfil it.

Expressed as a ratio, the $M I(m)$ for requirement $R_{m}$ :

$$
M I(n)=\frac{\text { The Mutation Value for } R_{m}}{\text { The maximum Mutation Value }}
$$

In the next section, we present how the RES-DIST technique uses the metrics Aptitude Index, Concordance Index, and Mutation Index to recommend merging or splitting of components.

\section{The RESP-DIST Technique}

Software design is about striking a balance (often a very delicate one!) between diverse factors that influence the functioning of a system. The ideas of aptitude, concordance, and mutation as outlined earlier are such factors we will consider now. The RESP-DIST technique builds on a LP formulation to maximize the Concordance Index across all components, for a given set of requirements, in a particular iteration of development, within the constraints of not increasing the number of components currently participating in the fulfilment of each requirement. Results from the LP solution are then examined in the light of the metric values and suggestions for merging or splitting components arrived at. (RESPDIST is the enhanced version of the COMP-REF technique we proposed in [7] - the latter only guided merging of components without addressing situations where components may require to be split.)

A new variable $a_{n}\left(a_{n} \in[0,1]\right)$ is introduced corresponding to each component $C_{n}, 1 \leq n \leq N$, where $N=$ the total number of components in the system. The values of $a_{n}$ are arrived at from the LP solution. Intuitively, $a_{n}$ for a component $C_{n}$ can be taken to indicate the extent to which $C_{n}$ contributes to maximizing the Concordance Index across all components. As we shall see later, the $a_{n}$ values will help us decide which components to merge.

The LP formulation can be represented as:

$$
\text { Maximize } \sum_{n=1}^{y} C I(n) a_{n}
$$

Subject to: $\forall R_{m} \in R e q, \sum_{n=1}^{y} a_{n} \leq p_{m} / N, a_{n}$ such that $C_{n} \in C S(m) \cdot p_{m}=$ $|C S(m)|$. (As defined in [6], the Component Set $C S(m)$ for a requirement $R_{m}$ is the set of components required to fulfil $R_{m}$.)

So, for a system with $x$ requirements and $y$ components, the objective function will have $y$ terms and there will be $x$ linear constraints.

The COMP-REF technique is summarized as: Given a set of requirements $R e q=\left\{R_{1}, \ldots, R_{x}\right\}$ and a set of components Comp $=\left\{C_{1}, \ldots, C_{y}\right\}$ fulfilling it in iteration $I_{z}$ of development, 
- STEP 0: Review Req and Comp for new or modified requirements and/or components compared to previous iteration.

- STEP 1: Calculate the Aptitude Index for each component.

- STEP 2: Calculate the Requirement Set for each component.

- STEP 3: Calculate the Concordance Index for each component.

- STEP 4: Formulate the objective function and the set of linear constraints.

- STEP 5: Solve the LP formulation for the values of $a_{n}$.

- STEP 6: For each component $C_{n}$, check:

- Condition 6.1: $a_{n}$ has a low value compared to that of other components? (If yes, implies $C_{n}$ is not contributing significantly to maximizing the concordance across the components.)

- Condition 6.2: $A I(n)$ has a low value compared to that of other components? (If yes, implies $C_{n}$ has to rely heavily on other components for delivering its core functionality.)

- STEP 7: If both conditions 6.1 and 6.2 hold TRUE, proceed to next step, else GO TO STEP 10

- STEP 8: For $C_{n}$, check:

- Condition 8.1: Upon merging $C_{n}$ with other components, in the resulting set $\tilde{C}$ omp of $q$ components (say), $C I(q) \neq 0$ for all $q$ ? (If yes, implies resulting set of $q$ components has more than one component).

- STEP 9: If condition 8.1 is TRUE, $C_{n}$ is a candidate for being merged.

- STEP 10: Let Comp $p^{\prime}$ denote the resulting set of components after above steps have been performed. For each component $C_{n^{\prime}}$ in $C o m p^{\prime}$ :

- 10.1 Calculate the average $M I(m)$ across all requirements in $R S\left(n^{\prime}\right)$. Let us call this $\bar{M} I(m)$.

- 10.2 Identify the requirement $R_{m}$ with the highest $M I(m)$ in $R S\left(n^{\prime}\right)$. Let us call this $M I(m)_{\text {highest }}$.

- STEP 11: For each component $C_{n^{\prime}}$, check:

- Condition 11.1: $A I\left(n^{\prime}\right)$ has a high value compared to that of other components? (If yes, implies component relies relatively less on other components for carrying out its primary responsibilities.)

- Condition 11.2: $C I\left(n^{\prime}\right)$ has a low value compared to that of other components? (If yes, implies component collaborates relatively less with other components for collectively delivering the system's functionality.)

- STEP 12: If both conditions 11.1 and 11.2 hold TRUE for component $C_{n^{\prime}}$, it is tending to be monolithic, doing all its activities by itself and collaborating less with other components. Thus the $C_{n^{\prime}}$ is a candidate for being split; proceed to next step, else GO TO STEP 14.

- STEP 13: Repeat STEPs 10 to 12 for all components of Comp'. For the component for which conditions 11.1 and 11.2 hold TRUE, choose the ones with the highest $\bar{M} I(m)$ and split each into two components, one with the requirement corresponding to the respective $M I(m)_{\text {highest }}$ and the other with remaining requirements (if any) of the respective Requirement Set. If the component was fulfilling only one requirement, the responsibility for fulfilling the requirement's functionality may now be delegated to two components.

- STEP 14: Wait for the next iteration of development. 


\section{Experimental Validation}

\subsection{Validation Strategy}

To explore whether or how dispersed development affects the distribution of responsibilities amongst software components, we have studied a number software projects, which vary significantly in their degrees of dispersion. The projects range from a single developer team, to an open source system being developed through a team whose members are located in different continents, a software system built by an in-house team of a large financial organization, and standalone utility systems built through remote collaboration. We discuss results from 5 such projects in the following subsections.

\subsection{Presentation of the Results}

Due to space constraints, we limit the detailed illustration of the application of RESP-DIST to one project in detail. The summary of all the validation scenarios are presented in Table 1 .

Table 2 gives metrics values and the LP solution for an iteration of Project A. Note: The project had 8 requirements: $R_{1}, R_{2}, R_{3}, R_{4}, R_{6}, R_{7}, R_{8}, R_{9}$ with requirement $R_{5}$ having been de-scoped in an earlier iteration of development. In the table Avg $M I(m)$ denotes $\bar{M} I(m)$ and $R_{m}{ }^{h}$ denotes the requirement $R_{m}$ with the highest $M I(m)$ in $R S\left(n^{\prime}\right) . M I(m)$ and $R_{m}{ }^{h}$ values are not applicable (NA) for $C_{4}$ since RESP-DIST recommends it to be merged as explained later.

From the design artefacts, we noted that $R_{1}$ needs components $C_{3}, C_{5}, C_{6}\left(p_{1}\right.$ $=3), R_{2}$ needs $C_{5}, C_{7}\left(p_{2}=2\right), R_{3}$ needs $C_{1}, C_{3}, C_{4}\left(p_{3}=3\right), R_{4}$ needs $C_{2}, C_{3}$ $\left(p_{4}=2\right), R_{6}$ needs $C_{1}, C_{2}, C_{6}\left(p_{6}=3\right), R_{7}$ needs $C_{2}, C_{6}\left(p_{7}=2\right), R_{8}$ needs $C_{7}$ $\left(p_{8}=1\right)$, and $R_{9}$ needs $C_{8}\left(p_{9}=1\right)$ for their respective fulfilments. Evidently, in this case $|W|=N=8$.

Based on the above, the objective function and the set of linear constraints was formulated as:

Maximize

$0.25 * a_{1}+0.25 * a_{2}+0.5 * a_{3}+0.13 * a_{4}+0.25 * a_{5}+0.25 * a_{6}+0.13 * a_{7}+0 . a_{8}$ Subject to

$$
\begin{aligned}
& a_{3}+a_{5}+a_{6} \leq 0.38 \\
& a_{1}+a_{3}+a_{4} \leq 0.38 \\
& a_{2}+a_{3} \leq 0.25 \\
& a_{1}+a_{2}+a_{6} \leq 0.38 \\
& a_{7} \leq 0.13 \\
& a_{8} \leq 0.13
\end{aligned}
$$

The linprog LP solver of MATLAB 1 was used to arrive at the values of $a_{n}$ in the Table 2, Let us examine how RESP-DIST can recommend the merging or splitting of components. Based on the $a_{n}$ values in Table2, evidently components $C_{2}, C_{4}, C_{6}$ have the least contribution to maximizing the objective function. So

\footnotetext{
${ }^{1}$ http://www.mathworks.com/
} 
Table 1. Experimental Validation: A Snapshot

\begin{tabular}{|c|c|c|c|}
\hline System & Scope and Technology & Salient Features & Findings \\
\hline Project A & $\begin{array}{l}\text { A } 5 \text { member team dispersed de- } \\
\text { velopment project }- \text { with } 1 \text { mem- } \\
\text { ber interfacing with the customer } \\
\text { and other members located in an- } \\
\text { other continent - to build an auto- } \\
\text { mated metrics driven tool to guide } \\
\text { the software development life cycle } \\
\text { activities. The system was released } \\
\text { as an open source product. }\end{array}$ & $\begin{array}{l}8 \text { requirements, } 8 \text { compo- } \\
\text { nents; system developed } \\
\text { using Java. }\end{array}$ & $\begin{array}{l}\text { RESP-DIST recommended } \\
1 \text { component be merged, } 1 \\
\text { component be split. Detailed } \\
\text { calculations are given later } \\
\text { in this section. }\end{array}$ \\
\hline Project B & $\begin{array}{l}\text { A } 2 \text { member team dispersed de- } \\
\text { velopment project - with virtual } \\
\text { collaboration between the team } \\
\text { members - to build a stan- } \\
\text { dalone utility to execute stan- } \\
\text { dard text classification algorithms } \\
\text { against bodies of text, allowing } \\
\text { for different algorithm implementa- } \\
\text { tions to be added, configured and } \\
\text { used. Among other uses, a spam de- } \\
\text { tection application can use this util- } \\
\text { ity to try out different detection al- } \\
\text { gorithms. }\end{array}$ & $\begin{array}{l}8 \text { requirements, } 7 \text { compo- } \\
\text { nents; system developed } \\
\text { using Java. The system } \\
\text { was selected from a com- } \\
\text { petition and integrated } \\
\text { in a broader application } \\
\text { framework. The develop- } \\
\text { ers had financial incen- } \\
\text { tives. }\end{array}$ & $\begin{array}{l}\text { RESP-DIST did not recom- } \\
\text { mended merging of any com- } \\
\text { ponents, but } 2 \text { components } \\
\text { could be split. }\end{array}$ \\
\hline Project $\mathrm{C}$ & $\begin{array}{l}\text { A } 2 \text { member team dispersed de- } \\
\text { velopment project - with virtual } \\
\text { collaboration between the team } \\
\text { members - to define, read, and } \\
\text { build an object representation of an } \\
\text { XML driven business work flow, al- } \\
\text { lowing manipulation and execution } \\
\text { of the workflow through a rich API } \\
\text { interface for the easy addition of } \\
\text { workflow operations. }\end{array}$ & $\begin{array}{l}11 \text { requirements, } 13 \text { com- } \\
\text { ponents; system devel- } \\
\text { oped using the .NET } \\
\text { platform. The system } \\
\text { was selected from a com- } \\
\text { petition and integrated } \\
\text { in a broader application } \\
\text { framework. The develop- } \\
\text { ers had financial incen- } \\
\text { tives. }\end{array}$ & $\begin{array}{l}\text { RESP-DIST recommended } \\
\text { merging of } 3 \text { components, } \\
\text { and splitting of } 2 \text { compo- } \\
\text { nents. }\end{array}$ \\
\hline Project D & $\begin{array}{l}\text { A } 6 \text { member team dispersed devel- } \\
\text { opment project - with the devel- } \\
\text { opers and customers spread across } \\
\text { two cities of the same country - to } \\
\text { develop an email response manage- } \\
\text { ment system for a very large finan- } \\
\text { cial company. The system allows for } \\
\text { emails from users across six prod- } \\
\text { uct segments to be processed and } \\
\text { placed in designated queues for cus- } \\
\text { tomer associates to respond, and } \\
\text { deliver the responded back to the } \\
\text { users within prescribed time limits. }\end{array}$ & $\begin{array}{l}5 \text { requirements; } 10 \\
\text { components; system } \\
\text { developed using Java, } \\
\text { Netscape Application } \\
\text { Server (NAS), and Lo- } \\
\text { tus Notes. Developers } \\
\text { worked on the system } \\
\text { as a part of their job } \\
\text { responsibilities. The } \\
\text { system has been running } \\
\text { for several years, with } \\
\text { around } 100,000 \text { users. }\end{array}$ & $\begin{array}{l}\text { RESP-DIST recommended } \\
\text { merging of } 1 \text { component, } \\
\text { and splitting of } 4 \text { compo- } \\
\text { nents. }\end{array}$ \\
\hline Project E & $\begin{array}{l}\text { A } 1 \text { member team project to build } \\
\text { a Web based banking application } \\
\text { which allowed users to check their } \\
\text { profile and account information, } \\
\text { send messages to the bank; and } \\
\text { administrators to manage user ac- } \\
\text { counts, transactions, and messages. }\end{array}$ & $\begin{array}{l}12 \text { requirements, } 28 \\
\text { components; system de- } \\
\text { veloped according to the } \\
\text { Model-View-Controller } \\
(\mathrm{MVC} \text { ) architectural } \\
\text { pattern with J2EE and } \\
\text { a Cloudscape database. }\end{array}$ & $\begin{array}{l}\text { Result from applying RESP- } \\
\text { DIST was inconclusive. }\end{array}$ \\
\hline
\end{tabular}


Table 2. Details for Project A

\begin{tabular}{|c|c|c|c|c|c|c|c|c|c|c|}
\hline$C_{m}$ & $R S(n)$ & Avg $M I(m)$ & $R_{m}{ }^{h}$ & $\alpha(n)$ & $\beta(n)$ & $\gamma(n)$ & $A I(n)$ & $X(n)$ & $C I(n)$ & $a_{n}$ \\
\hline$C_{1}$ & $R_{3}, R_{6}$ & 0 & - & $C_{1}$ & $C_{3}, C_{5}, C_{7}$ & - & 0.25 & 2 & 0.25 & 0.21 \\
\hline$C_{2}$ & $R_{4}, R_{7}$ & 0 & - & $C_{2}$ & $C_{3}, C_{7}$ & $C_{6}$ & 0.2 & 2 & 0.25 & 0.08 \\
\hline$C_{3}$ & $R_{1}, R_{3}, R_{4}, R_{6}$ & 0.17 & $R_{1}$ & $C_{3}$ & $C_{1}, C_{5}, C_{7}$ & - & 0.25 & 4 & 0.5 & 0.17 \\
\hline$C_{4}$ & $R_{3}$ & $\mathrm{NA}$ & $\mathrm{NA}$ & $C_{4}$ & $C_{3}, C_{5}$ & - & 0.33 & 1 & 0.13 & 0 \\
\hline$C_{5}$ & $R_{1}, R_{2}$ & 0.5 & $R_{1}$ & $C_{5}$ & $C_{1}$ & - & 0.5 & 2 & 0.25 & 0.12 \\
\hline$C_{6}$ & $R_{1}, R_{7}$ & 0.34 & $R_{1}$ & $C_{6}$ & $C_{2}, C_{7}$ & - & 0.33 & 2 & 0.25 & 0.09 \\
\hline$C_{7}$ & $R_{2}, R_{8}$ & 0.5 & $R_{8}$ & $C_{7}$ & - & - & 1 & 1 & 0.13 & 0.13 \\
\hline$C_{8}$ & $R_{9}$ & 1 & $R_{9}$ & $C_{8}$ & - & - & 1 & 0 & 0 & 0.105 \\
\hline
\end{tabular}

the tasks performed by these components may be delegated to other components. However, as mandated by RESP-DIST, another factor needs be taken into account before merging. How self-sufficient are the components that are sought to be merged? We thus turn to the $A I(n)$ values for the components in Table 2. We notice, $A I(2)=0.2, A I(4)=0.33, A I(6)=0.33$. Out of these, $C_{4}$ is contributing nothing to maximizing concordance $\left(a_{4}=0\right)$, and its $A I(n)$ value is not very high either (0.33 on a scale of 1$)$. So $a_{4}$ can be merged with other components. Now we check for the highest $\bar{M} I(m)$, which corresponds to $C_{8} . C_{8}$ also has a high $A I(8)$ value of 1 and a low $C I(8)$ value of 0 . Thus $C_{8}$ is trying to do all its task by itself, without collaborating with other components - this is indeed a candidate for splitting. The $R_{m}$ with the highest $M I(m)$ in $R S(8)$ is $R_{9}$ - in fact $R_{9}$ is the only requirement in this particular case fulfilled by $C_{8}$. So RESP-DIST recommends $C_{8}$ be split into two components, each fulfilling a part of $R_{9}$. Relating the recommendations to the actual components and requirements, we find that $C_{4}$ is an utility component in charge of carrying out some numerical calculations; whose tasks can very well be re-assigned to components which contain the business logic behind the calculations. On the other hand, $R_{9}$ is a requirement for extracting data from design artefacts. This is certainly a requirement of very large sweep and one likely to change frequently, as the data needs of the users change. Thus it is justifiable to have $R_{9}$ fulfilled by more than one component, to be able to better localize the effects of potential changes in this requirement. Figure 1 summarizes these discussions, indicating merging for $C_{4}$ and splitting for $C_{8}$.

\subsection{Interpretation of the Results}

We examined the factors of dispersed development that could potentially affect the outcome of applying the RESP-DIST technique on these projects.

The Agile Manifesto lists the principles behind agile software development - methodologies being increasingly adopted for delivering quality software in large and small projects in the industry, including those utilizing dispersed development [15. The Manifesto mentions the following among a set of credos: "The most efficient and effective method of conveying information to and within a development team is face-to-face conversation", and "Business people and 


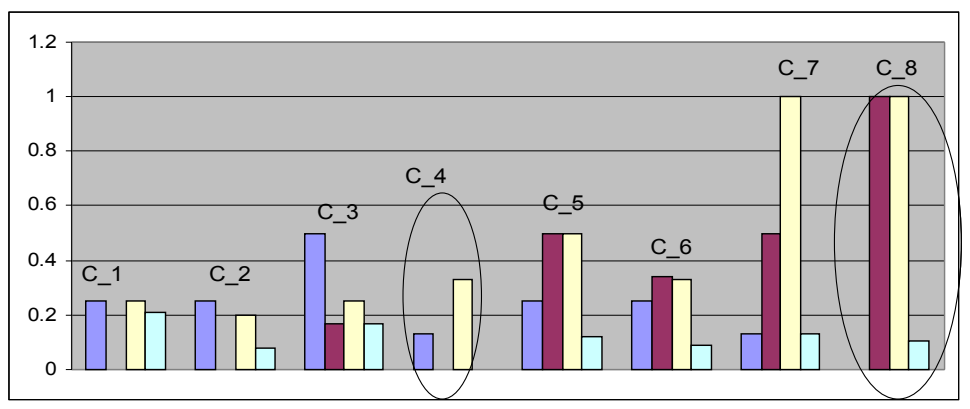

Fig. 1. Values of $a_{n}, A I(n), \bar{M} I(m)$ and $C I(n)$ corresponding to the components $C_{1}, \ldots, C_{8}$ for Project A. The RESP-DIST technique suggests merging for $C_{4}$ and splitting for $C_{8}$ - detailed discussion in section 6.3.

developers must work together daily throughout the project" 2. Evidently, the very nature of dispersed development precludes this kind of interaction between those who commission and use a software system (these two groups may be identical or different, they are often clubbed together as customers) and those who develop it, that is, the developers.

We identify the key drivers of the effects of dispersed development on software design as locational asynchrony (LA), and perceptional asynchrony (PA). LA and PA may exist between customers and developers or within the development team. Locational asynchrony arises from factors like differences in geography and time zones. An example of LA would be the difficulty in explaining a simple architectural block diagram over email or telephone conversation, which can be easily accomplished with a white board and markers in a room of people (something similar to the consequence of distance highlighted in [10]). Perceptional asynchrony tends to be more subtle, and is caused by the complex interplay of stakeholder interests that dispersed development essentially entails. For example, in dispersed development scenarios, developers who have no direct interaction with the customer often find it hard to visualize the relevance of the module they are working on in the overall business context of the application - this is a manifestation of PA. With reference to Table 1, Project A has high LA but moderate PA; Projects B and C have moderate LA but high PA; Project D has moderate LA and low PA, while Project E has low LA and PA.

Apparently, there is no clear trend in the recommendations from RESP-DIST by way of merging or splitting components in Table 1 that suggests locational asynchrony or perceptional asynchrony have noticeable impact on how responsibilities are delegated. However, Projects B and C have a higher requirement to component ratio compared to others. This not only influences the way RESPDIST runs on these projects but also indicates that moderate to high perceptional asynchrony may lead to a more defensive analysis of requirements - being

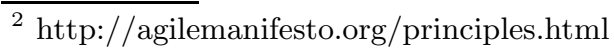


relatively unsure of the customers' intents developers are more comfortable dealing with finer grained requirements. The inconclusiveness of RESP-DIST's recommendation for Project E is also interesting. Project E's scenario represents by far the most controlled conditions of development amongst all the projects studied. It was developed by a single developer - a software engineer with more than $5+$ years of industry experience - who had the mandate to refine the responsibility delegations amongst components repeatedly until the system delivered as expected. So naturally, RESP-DIST did not have much scope for suggesting merging or splitting of components. Also, compared to other projects Project E had a relatively unrelated set of requirements and relatively high number components with uniformly distributed responsibilities. Thus from the results related to Project A to D, RESP-DIST is seen to work best on a small set of closely related requirements and components. For a system with many requirements and components, it can be applied separately on subsystems that constitute the whole system.

\section{Related Work}

Freeman's paper, Automating Software Design, is one of the earliest expositions of the ideas and issues relating to design automation [9]. Karimi et al. 14] report their experiences with the implementation of an automated software design assistant tool. Ciupke presents a tool based technique for analyzing legacy code to detect design problems [3. Jackson's Alloy Analyzer tool employs "automated reasoning techniques that treat a software design problem as a giant puzzle to be solved" 12 .

Collaboration platforms for offshore software development are evaluated in 17. Shami et al. simulate dispersed development scenarios 20 and a research agenda for this new way of software building is presented in [19].

Herbsleb and Grinter in their papers [10, [11 have taken a more social view of distributed software development. In terms of Conway's Law - organizations which design systems are constrained to produce designs which are copies of the communication structures of these organizations 4] - Herbsleb and Grinter seek to establish the importance of the match between how software components collaborate and how the members of the teams that develop the software components collaborate.

\section{Open Issues and Future Work}

From the interpretation of the case study results, it is apparent the recommendations of merging or splitting components from applying the RESP-DIST technique are not significantly influenced by the degree of dispersion in a project's development scenario in terms of their location or perceptional asynchronies. However, factors other than locational or perceptional asynchrony may also 
stand to affect the delegation of responsibilities in some dispersed development projects. In future work we plan to develop mechanisms to investigate such situations.

The case studies we presented in this paper range from 1 member to 6 member development teams, 5 to 12 requirements, and 7 to 28 components. Evidently, these are small to medium projects. We expect the execution of the RESPDIST technique to scale smoothly to larger systems - more requirements and components will only mean more terms and linear constraints, which can be handled easily by automated LP solvers. However, the ramifications of larger systems on the dynamics of dispersed development is something which can only be understood by further case studies, some of which are ongoing.

We also plan to fine-tune the RESP-DIST technique by studying more projects across a diverse range of technology and functional area. The applicability of techniques like RESP-DIST are highly enhanced with tool support. We are working on an automated tool that will take in design artefacts and/or code as input, apply RESP-DIST and suggest the merging or splitting of relevant component.

\section{Conclusion}

In this paper, we examined whether and how offshore and outsourced development influences the delegation of responsibilities to software components. We applied the RESP-DIST technique - which uses the metrics Aptitude Index, Mutation Index and Dependency Index, and a linear programming based algorithm to recommend reorganization of the responsibilities of a software system's components through merging or splitting - on a range of software projects that embody varying degrees of offshore and outsourced development. It appears that two aspects of offshore and outsourced development - what we call as locational asynchrony and perceptional asynchrony - do not have significant effect on how responsibilities are distributed in the projects studied. However these factors may influence the way requirements are abstracted by the development team, which in turn can influences the application of the RESP-DIST technique. We plan to extend our work in refining the RESP-DIST technique by conducting further case studies. We are also working on developing a software tool to automate the application of the RESP-DIST technique.

\section{Acknowledgements}

We wish to thank Sean Campion, Project Manager at TopCoder Inc.; Dr Animikh Sen, Executive Director of Strategic Planning and Program Development at Boca Raton Community Hospital; Kshitiz Goel, Pooja Mantri, Prerna Gandhi, and Sidharth Malhotra, graduate students at Symbiosis Center for Information Technology for their help with acquiring and analyzing some of the case study information. We would also like to thank the anonymous reviewers for their helpful comments and criticism. 


\section{References}

1. Bieman, J.M., Ott, L.M.: Measuring functional cohesion. IEEE Trans. Softw. Eng. 20(8), 644-657 (1994)

2. Booch, G., Rumbaugh, J., Jacobson, I.: The Unified Modeling Language User Guide, 2nd edn. Addison-Wesley, Reading (2005)

3. Ciupke, O.: Automatic detection of design problems in object-oriented reengineering. In: TOOLS 1999: Proceedings of the Technology of Object-Oriented Languages and Systems, Washington, DC, USA, p. 18. IEEE Computer Society, Los Alamitos (1999)

4. Conway, M.: How do committees invent? Datamation Journal, 28-31 (April 1968)

5. Datta, S.: Agility measurement index: a metric for the crossroads of software development methodologies. In: ACM-SE 44: Proceedings of the 44th annual southeast regional conference, pp. 271-273. ACM Press, New York (2006)

6. Datta, S., van Engelen, R.: Effects of changing requirements: a tracking mechanism for the analysis workflow. In: SAC 2006: Proceedings of the 2006 ACM symposium on Applied computing, pp. 1739-1744. ACM Press, New York (2007)

7. Datta, S., van Engelen, R.: Comp-ref: A technique to guide the delegation of responsibilities to components in software systems. In: Fiadeiro, J.L., Inverardi, P. (eds.) FASE 2008. LNCS, vol. 4961, pp. 332-346. Springer, Heidelberg (2008)

8. Dhama, H.: Quantitative models of cohesion and coupling in software. In: Selected papers of the sixth annual Oregon workshop on Software metrics, pp. 65-74. Elsevier Science Inc., Amsterdam (1995)

9. Freeman, P.: Automating software design. In: DAC 1973: Proceedings of the 10th workshop on Design automation, Piscataway, NJ, USA, pp. 62-67. IEEE Press, Los Alamitos (1973)

10. Herbsleb, J.D., Grinter, R.E.: Architectures, coordination, and distance: Conway's law and beyond. IEEE Softw. 16(5), 63-70 (1999)

11. Herbsleb, J.D., Grinter, R.E.: Splitting the organization and integrating the code: Conway's law revisited. In: ICSE 1999: Proceedings of the 21st international conference on Software engineering, pp. 85-95. IEEE Computer Society Press, Los Alamitos (1999)

12. Jackson, D.: Software Abstractions: Logic, Language and Analysis. MIT Press, Cambridge (2006)

13. Jacobson, I., Booch, G., Rumbaugh, J.: The Unified Software Development Process. Addison-Wesley, Reading (1999)

14. Karimi, J., Konsynski, B.R.: An automated software design assistant. IEEE Trans. Softw. Eng. 14(2), 194-210 (1988)

15. Kornstadt, A., Sauer, J.: Mastering dual-shore development - the tools and materials approach adapted to agile offshoring. In: Meyer, B., Joseph, M. (eds.) SEAFOOD 2007. LNCS, vol. 4716, pp. 83-95. Springer, Heidelberg (2007)

16. Larman, C.: Applying UML and Patterns. Prentice Hall, Englewood Cliffs (1997)

17. Rodriguez, F., Geisser, M., Berkling, K., Hildenbrand, T.: Evaluating collaboration platforms for offshore software development scenarios. In: Meyer, B., Joseph, M. (eds.) SEAFOOD 2007. LNCS, vol. 4716, pp. 96-108. Springer, Heidelberg (2007) 
18. Rumbaugh, J., Jacobson, I., Booch, G.: The Unified Modeling Language Reference Manual, 2nd edn. Addison-Wesley, Reading (2005)

19. Sengupta, B., Chandra, S., Sinha, V.: A research agenda for distributed software development. In: ICSE 2006: Proceeding of the 28th international conference on Software engineering, pp. 731-740. ACM, New York (2006)

20. Shami, N.S., Bos, N., Wright, Z., Hoch, S., Kuan, K.Y., Olson, J., Olson, G.: An experimental simulation of multi-site software development. In: CASCON 2004: Proceedings of the 2004 conference of the Centre for Advanced Studies on Collaborative research, pp. 255-266. IBM Press (2004) 